\title{
Synthesis and Properties of 1,3-Disubstituted Ureas and Their Isosteric Analogs Containing Polycyclic Fragments: VII. ${ }^{1}$ Synthesis and Properties 1-[(Adamantan-1-yl)methyl]- 3-(fluoro, chlorophenyl) Ureas
}

\author{
D. V. Danilov ${ }^{a}$, V. S. D’yachenko ${ }^{a, b}$, Y. P. Kuznetsov ${ }^{a}$, V. V. Burmistrov ${ }^{a, b}$, and G. M. Butov ${ }^{a, b, *}$ \\ ${ }^{a}$ Volgograd State Technical University (VSTU), Volgograd 400005 Russia \\ ${ }^{b}$ Volzhskii Polytechnic Institute, Branch of VSTU, Volzhskii, 404121 Russia \\ *e-mail: butov@volpi.ru
}

Received October 22, 2020; revised October 29, 2020; accepted October 30, 2020

\begin{abstract}
A series of 1,3-disubstituted ureas containing a lipophilic adamantane moiety tethered to the ureido group by a methylene bridge were synthesized by the reaction of 1-(isocyanatomethyl)adamantane with monohalo- and dihaloanilines in yields of up to $92 \%$. The synthesized ureas are target oriented inhibitors of human soluble epoxide hydrolase (sEH).
\end{abstract}

Keywords: adamantane, isocyanate, urea, halogen-containing anilines, soluble epoxide hydrolase, sEH

DOI: $10.1134 / \mathrm{S} 1070428021020020$

\section{INTRODUCTION}

Urea derivatives are known to exhibit broadspectrum biological activity. For example, ethyl 2-(4-R-1,4-diazepane-1-carboxamido)benzoates are potential antiviral RNA viruses, such as SARS-CoV, HIV-1, and ARVI [2]. Such compounds as 1-(3-chloro4-methylphenyl)-3-(4-phenylbutan-2-yl)urea, which contain halogenated benzene moieties, are active against Staphylococcus aureus and Klebsiella pneumoniae (Friedlander's wand). As known, some Klebsiella bacterial strains are fully resistant to antibacterial drugs, as well as to the anthrax causative agent, Bacillus anthracis [3].

Proceeding with the research on the synthesis of soluble epoxide hydrolase (sEH, E.C. 3.3.2.10), an enzyme of the arachidonic cascade enzyme [4-7], involved in the metabolic transformation of epoxy fatty acids to vicinal diols, we performed targeted modification of the lipophilic part of inhibitor molecules. It was found that the separation of the adamantyl from ureido/ thioureido group with a methylene bridge enhances the inhibitory activity against $\mathrm{sEH}$ by a factor of $2-4$, and also has a positive effect on water solubility [8-10].

\footnotetext{
1 For communication VI, see [1].
}

The choice of the structure of the starting compounds is explained by that they contain both an aromatic fragment, which can enter hydrophobic interactions with the target, and fluorine and chlorine atoms, which are H-bond acceptors. The presence of such groups can ensure additional bonding in the enzyme active site and thus enhance inhibitory activity and affect the water solubility and melting point of the synthesized compounds.

\section{RESULTS AND DISCUSSION}

The well-known Curtius synthesis of 1-(isocyanatomethyl)adamantane (3) from (adamantan-1-yl)acetic acid involves the intermediate synthesis of (adamantan-1-yl)acetyl chloride, followed by the reaction of the latter with sodium azide [11]. A significant disadvantage of this method is that it makes use of a toxic thionyl chloroide and an explosive sodium azide. Therefore, in this work, isocyanate $\mathbf{3}$ was synthesized by a one-step procedure using diphenylphosphoryl azide (DPPA) as a source of the acylazido group (Scheme 1).

To this end, we prepared (adamantan-1-yl)acetic acid (2) from 1-bromoadamantane (1) in a yield of 93\%. Acid 2 was further treated with equimolar amounts of DPPA 


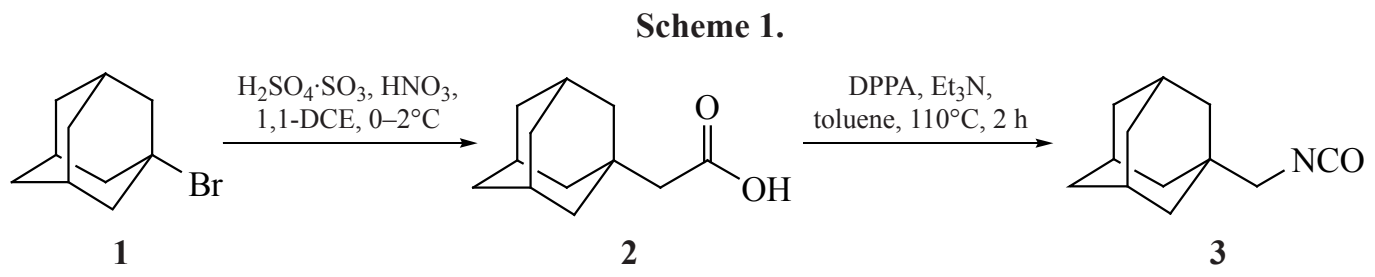

and $\mathrm{Et}_{3} \mathrm{~N}$ to obtain 1-(isocyanatomethyl)adamantane (3) in a yield of $85 \%$.

Isocyanate 3 was reacted with mono- and dihaloanilines $\mathbf{4 a - 4 o}$ to prepare 1,3-disubstituted ureas 5a-5o (Scheme 2).

The synthesis was performed in dry DMF for $12 \mathrm{~h}$ at room temperature. The yields were $10-92 \%$.

The structure of the synthesized compounds was conformed by NMR spectroscopy and mass spectrometry. The ${ }^{1} \mathrm{H}$ NMR spectra contain two characteristic urea NH proton signals. The signal at 5.68-6.81 ppm corresponds to the proton of the $\mathrm{NH}$ group proximate to the adamantyl fragment, whereas the signal at $7.75-8.64 \mathrm{ppm}$, to the proton of the $\mathrm{NH}$ group linked to the aromatic ring.

Analysis of the ${ }^{19} \mathrm{~F}$ NMR signals showed the dependence of the shifts of the signals of the $\mathrm{F}$ atom on their position in the aromatic ring. Thus, the signals of the $\mathrm{F}$ atoms in the $\mathrm{R}^{1}$ positions of the aromatic rings appear at -119.08 to $-133.10 \mathrm{ppm}(\mathbf{5} \mathbf{c}, \mathbf{5 d}, \mathbf{5 f}, \mathbf{5 k}, \mathbf{5 m}$, and $5 n$ ); the signals of $F$ atoms in the $R^{2}$ and $R^{4}$ are at -110.01 to $-137.77 \mathrm{ppm}(\mathbf{5 a}, \mathbf{5 e}-\mathbf{5 g}$, and $\mathbf{5 l})$; and the signals of the $\mathrm{F}$ atoms in the $\mathrm{R}^{3}$ and $\mathrm{R}^{5}$ positions, at -120.52 to $148.57 \mathrm{ppm}(\mathbf{5 b}, \mathbf{5 c}, \mathbf{5 e}, \mathbf{5 i}$, and $\mathbf{5 j})$ and -116.70 to $-119.08 \mathrm{ppm}(\mathbf{5 d})$, respectively. In the ${ }^{19} \mathrm{~F}$
NMR spectrum of compound 5o, the trifluoromethyl fluorine atoms give one signal at $-61.33 \mathrm{ppm}$. The chemical shifts of the fluorine atoms are also dependent on whether there are other $\mathrm{F}$ and $\mathrm{Cl}$ substituents in the aromatic ring.

The yields of ureas were directly related to the substrate structure. The highest yields (92\%) were characteristic of the ureas containing one $\mathrm{F}$ atom in the aromatic ring. With the substrates containing two $\mathrm{F}$ atoms, the yields decreased to $10-82 \%$. The lowest yield was obtained with urea $\mathbf{5 d}(10 \%)$, in while the $\mathrm{F}$ atoms are ortho to each other. The properties of the synthesized 1,3-disubstituted ureas 5a-5o are listed in the Table 1.

The calculated lipophilicity coefficients $\log P$ of the synthesized compounds span the range 4.52-5.25, which is higher, on average, by 0.09 compared to those of the related compounds derived from unsubstituted 1-(isocyanato)adamantane [1] and lower by 0.17 compared to those of the related compounds derived from 1-(isocyanatoethyl)adamantane [12]. The $\log P$ values of ureas $\mathbf{5 a}-\mathbf{5 g}$, which contain no other substituents in the aromatic ring but fluorines, vary between 4.52 and 4.64, and, therefore, comply with the Lipinski's rule [13]. The replacement of the fluorine substituent in the aromatic ring by chlorine increases the lipophilicity coefficient to 5.04-5.25. This change

Scheme 2.

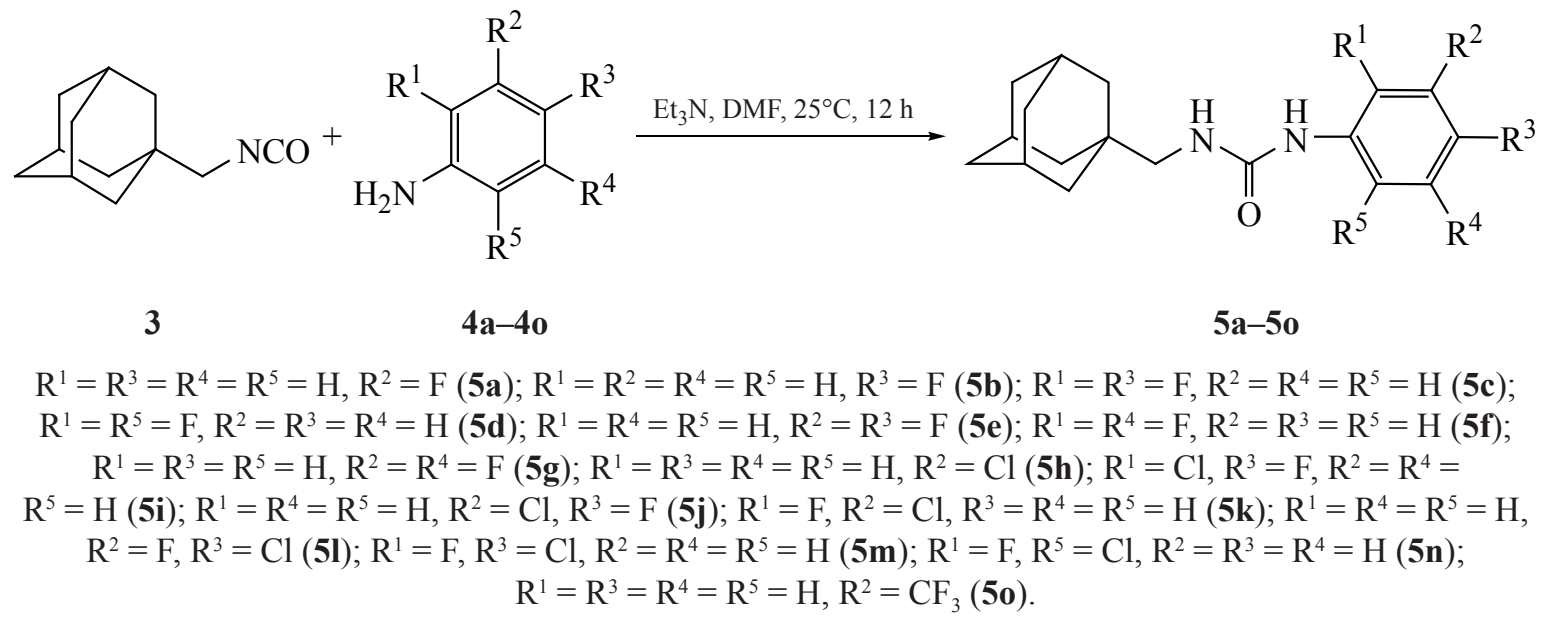


is likely to be associated with the lower electrophilicity of $\mathrm{Cl}$ composed to $\mathrm{F}$. The $\mathrm{C}-\mathrm{F}$ bond conjugated with a double bond acquires a double-bond character, as a resul of which the radius of the covalent bond becomes even smaller, and, as a result, spatially the fluorine atom becomes almost indistinguishable from the hydrogen atom [14].
The melting points of the synthesized ureas, which contain a methylene bridge between the adamantane and urea fragments are lower by $49-122^{\circ} \mathrm{C}$ compared to those of the related compounds derived from unsubstituted 1-(isocyanato)adamantane. Lower melting points are a positive property of inhibitors, because this facilitates the manufacturing of dosage forms.

Table 1. Lipophilicity coefficients, melting points, and yields of synthesized compounds $\mathbf{5 a}-\mathbf{5 0}$

\begin{tabular}{|c|c|c|c|c|c|}
\hline Compound no. & Structure & $\mathrm{Mr}$ & $\log P^{\mathrm{a}}$ & $\mathrm{mp},{ }^{\circ} \mathrm{C}$ & Yield, \% \\
\hline $5 a$ & & 302 & 4.52 & $87-88$ & 92 \\
\hline $5 b$ & & 302 & 4.55 & $170-171$ & 92 \\
\hline $5 c$ & & 320 & 4.64 & $167-168$ & 74 \\
\hline $5 d$ & & 320 & 4.61 & $182-183$ & 10 \\
\hline $5 e$ & & 320 & 4.64 & $142-143$ & 70 \\
\hline $5 f$ & & 320 & 4.64 & $92-93$ & 82 \\
\hline $5 g$ & & 320 & 4.64 & $124-125$ & 70 \\
\hline
\end{tabular}


Table 1. (Contd.)

\begin{tabular}{|c|c|c|c|c|c|}
\hline Compound no. & Structure & $\mathrm{Mr}$ & $\log P^{\mathrm{a}}$ & $\mathrm{mp},{ }^{\circ} \mathrm{C}$ & Yield, \% \\
\hline $5 \mathrm{~h}$ & & 319 & 5.04 & $163-164$ & 92 \\
\hline $5 \mathbf{i}$ & & 337 & 5.15 & $161-162$ & 72 \\
\hline $5 \mathbf{j}$ & & 337 & 5.15 & $115-116$ & 73 \\
\hline $5 \mathbf{k}$ & & 337 & 5.13 & $120-122$ & 85 \\
\hline 51 & & 337 & 5.15 & $102-105$ & 76 \\
\hline $5 \mathrm{~m}$ & & 337 & 5.15 & $109-110$ & 76 \\
\hline $5 n$ & & 337 & 5.13 & $113-114$ & 79 \\
\hline 50 & & 352 & 5.25 & $153-155$ & 71 \\
\hline
\end{tabular}

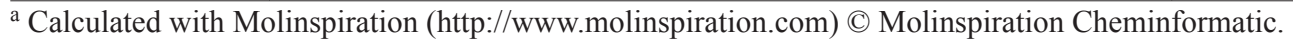

\section{EXPERIMENTAL}

3-Chloroaniline (99\%, CAS 108-42-9), 3-chloro-4fluoroaniline (98\%, CAS 367-21-5), 3-(trifluoromethyl)aniline $(\geq 99 \%$, CAS 98-16-8), triethylamine (BioUltra $\geq 99.5 \%$, CAS 121-44-8), and DMF NMR spectrum (Anhydrous 99.8\%, CAS 68-12-2) were purchased from Sigma-Aldrich; 3-fluoroaniline (99\%, CAS 372-19-0), 4-fluoroaniline(99\%,CAS371-40-4),2,4-difluoroaniline 
(99\%, CAS 367-25-9), 2,6-difluoroaniline (98\%, CAS 5509-65-9), 3,4-difluoroaniline (98+\%, CAS 386311-4), 2,5-difluoroaniline (99\%, CAS 367-30-6), 3,5-difluoroaniline (98\%, CAS 372-39-4), 2-chloro4-fluoroaniline (97\%, CAS 2106-02-7), 3-chloro-4fluoroaniline (98\%, CAS 367-21-5), 3-chloro-2-fluoroaniline (97\%, CAS 2106-04-9), 4-chloro-2-fluoroaniline (98\%, CAS 57946-56-2), and 2-chloro-6-fluoroaniline (97\%, CAS 363-51-9) were obtained from Alfa Aesar and used as received.

The structure of the synthesized compounds was confirmed by ${ }^{1} \mathrm{H},{ }^{13} \mathrm{C}$, and ${ }^{19} \mathrm{~F}$ NMR spectroscopy, gas chromatography-mass spectrometry, and elemental analysis. The mass spectra were run on an Agilent GC 7820/MSD 5975 system and an Advion Expression compact mass spectrometer in the full scan mode (ESI). The ${ }^{1} \mathrm{H},{ }^{13} \mathrm{C}$, and ${ }^{19} \mathrm{~F}$ NMR spectra were measured on a Bruker Avance 600 spectrometer in DMSO- $d_{6}$; internal reference $\mathrm{SiMe}_{4}$. The elemental analyses were obtained on a Perkin-Elmer Series II 2400 CHNS/O analyzer.

(Adamantan-1-yl)acetic acid (2). A three-necked reactor placed in an ice bath and equipped with a thermometer, a dropping funnel, and an overhead stirrer was charged, with stirring, with $540 \mathrm{~mL}$ of $98 \% \mathrm{H}_{2} \mathrm{SO}_{4}$ and $12.5 \mathrm{~mL}$ of $65 \% \mathrm{HNO}_{3}$. The mixture was cooled to $0-2^{\circ} \mathrm{C}$, after which $100 \mathrm{~g}(0.475 \mathrm{~mol})$ of 1-bromoadamantane was added and then $271 \mathrm{~mL}$ ( $3.487 \mathrm{~mol}$ ) of vinylidene chloride was added dropwise over the course of $1.5 \mathrm{~h}$, maintaining the temperature at $0-2^{\circ} \mathrm{C}$. The mixture was stirred at the same temperature for another $1 \mathrm{~h}$ and poured onto ice. After the ice had melted, the product was filtered off, washed with distilled water, and purified by reprecipitation. Yield 83.9 g (93\%), mp $135-136^{\circ} \mathrm{C}$. Mass spectrum, $m / z$ $\left(I_{\text {rel }}, \%\right): 194(5)[M]^{+}, 149(22.0)[M-\mathrm{COOH}]^{+}, 135$ (100.0) $[\mathrm{Ad}]^{+}$. Found, \%: C 74.11; $\mathrm{H}$ 9.38. $\mathrm{C}_{12} \mathrm{H}_{18} \mathrm{O}_{2}$. Calculated, \%: C 74.19; H 9.34. M 194.23.

1-(Isocyanatomethyl)adamantane (3). Diisopropyl fluorophosphate, $5.6 \mathrm{~mL}(25.8 \mathrm{mmol})$, was added dropwise over the course of $30 \mathrm{~min}$ to a mixture of $5 \mathrm{~g}(25.8 \mathrm{mmol})$ of acid 2 and $3.6 \mathrm{~mL}(26.0 \mathrm{mmol})$ of triethylamine in $100 \mathrm{~mL}$ of dry toluene. The reaction mixture was then heated under reflux for $30 \mathrm{~min}$ until nitrogen no longer evolved. Toluene was evaporated, and the product was extracted with dry diethyl ether. Yield $4.18 \mathrm{~g}(85 \%)$, oily liquid. ${ }^{1} \mathrm{H}$ NMR spectrum (DMSO- $\left.d_{6}\right), \delta$, ppm: $1.50-1.95$ m (15H, Ad), 2.98 s $(2 \mathrm{H}$,
$\left.\mathrm{CH}_{2}\right)$. Mass spectrum, $\mathrm{m} / \mathrm{z}\left(\mathrm{I}_{\text {rel }}, \%\right): 191.2(6.0)[\mathrm{M}]^{+}$, $149.2(30.0)\left[M-\mathrm{NCO}^{+}, 135(100.0)[\mathrm{Ad}]^{+}\right.$. Found, \%: C 75.33; $\mathrm{H} 8.99 ; \mathrm{N} 7.35 . \mathrm{C}_{12} \mathrm{H}_{17} \mathrm{NO}$. Calculated, \%: C 75.35; H 8.96; N 7.32. M 191.27.

1-(Adamantan-1-ylmethyl)-3-(3-fluorophenyl)urea (5a). 3-Fluoroaniline, $0.145 \mathrm{~g}(1.3 \mathrm{mmol})$, and $0.2 \mathrm{~mL}$ of triethylamine were added to $0.25 \mathrm{~g}$ (1.3 mmol) of isocyanate 3 in $5 \mathrm{~mL}$ of dry DMF. The reaction mixture was allowed to stand for $12 \mathrm{~h}$ at room temperature, after which $6 \mathrm{~mL}$ of $1 \mathrm{~N} \mathrm{HCl}$ was added, and the resulting mixture was stirred for $1 \mathrm{~h}$. The white precipitate that formed was filtered off, washed with water, and recrystallized from ethanol. Yield $0.364 \mathrm{~g}$ (92\%), mp 87-88 ${ }^{\circ} \mathrm{C} .{ }^{1} \mathrm{H}$ NMR spectrum (DMSO- $d_{6}$ ), $\delta$, ppm: 1.17-1.20 t (1H, Ad, $\left.J_{1} 7.3, J_{2} 7.3 \mathrm{~Hz}\right), 1.43 \mathrm{~d}$ $(1 \mathrm{H}, \mathrm{Ad}, J 2.9 \mathrm{~Hz}), 1.47 \mathrm{~d}(6 \mathrm{H}, \mathrm{Ad}, J 2.9 \mathrm{~Hz}), 1.66 \mathrm{q}$ (4H, Ad, $\left.J_{1} 11.1, J_{2} 11.9 \mathrm{~Hz}\right), 1.96$ br.s $(3 \mathrm{H}, \mathrm{Ad}), 2.82 \mathrm{~d}$ $\left(2 \mathrm{H}, \mathrm{CH}_{2}, J 6.0 \mathrm{~Hz}\right), 6.17 \mathrm{t}\left(1 \mathrm{H}, \mathrm{N} \underline{\mathrm{H}}-\mathrm{CH}_{2}-\mathrm{Ad}, J_{1} 6.1\right.$, $\left.J_{2} 6.1 \mathrm{~Hz}\right), 6.67$ t.d.d $\left(1 \mathrm{H}, 4-\mathrm{H}_{\text {arom }}, J_{1} 8.5, J_{2} 2.6, J_{3}\right.$ $0.9 \mathrm{~Hz}), 7.01$ d.d.d $\left(1 \mathrm{H}, 5-\mathrm{H}_{\text {arom }}, J_{1} 8.2, J_{2} 2.0, J_{3}\right.$ $0.9 \mathrm{~Hz}), 7.22$ t.d $\left(1 \mathrm{H}, 6-\mathrm{H}_{\text {arom }}, J_{1} 8.2, J_{2} 8.2, J_{3} 6.9 \mathrm{~Hz}\right)$, 7.45 d.t (1H, 2- $\left.\mathrm{H}_{\text {arom }}, J_{1} 12.3, J_{2} 2.3 \mathrm{~Hz}\right), 8.64 \mathrm{~s}(1 \mathrm{H}$, $\mathrm{N} \underline{\mathrm{H}}-\mathrm{Ph}) .{ }^{19} \mathrm{~F}$ NMR spectrum (DMSO- $\left.d_{6}\right), \delta$, ppm: -112.49. Mass spectrum, $m / z\left(I_{\text {rel }}, \%\right): 303$ (77.4) $[M+$ $\mathrm{H}]^{+}, 605$ (12.3) $[2 M+\mathrm{H}]^{+}$. Found, \%: C 71.47; H 7.69; F 6.25; N 9.29. $\mathrm{C}_{18} \mathrm{H}_{23} \mathrm{FN}_{2} \mathrm{O}$. Calculated, \%: C 71.50; H 7.67; F 6.28; N 9.26. M 302.39.

1-(Adamantan-1-ylmethyl)-3-(4-fluorophenyl)urea (5b) was prepared similarly to compound 5a, from $0.25 \mathrm{~g}$ of isocyanate 3 and $0.145 \mathrm{~g}$ of 4-fluoroaniline. Yield $0.360 \mathrm{~g}(92 \%), \mathrm{mp} 170-171^{\circ} \mathrm{C} .{ }^{1} \mathrm{H}$ NMR spectrum (DMSO- $\left.d_{6}\right), \delta$, ppm: $1.47 \mathrm{~d}(6 \mathrm{H}, \mathrm{Ad}, J 2.9 \mathrm{~Hz})$, $1.66 \mathrm{q}\left(6 \mathrm{H}, \mathrm{Ad}, J_{1} 12.6, J_{2} 12.6 \mathrm{~Hz}\right), 1.96$ br.s $(3 \mathrm{H}$, Ad), $2.81 \mathrm{~d}\left(2 \mathrm{H}, \mathrm{CH}_{2}, J 6.1 \mathrm{~Hz}\right), 6.01 \mathrm{t}\left(1 \mathrm{H}, \mathrm{N} \underline{\mathrm{H}}-\mathrm{CH}_{2}-\right.$ $\left.\mathrm{Ad}, J_{1} 6.1, J_{2} 6.1 \mathrm{~Hz}\right), 7.00-7.07 \mathrm{~m}\left(2 \mathrm{H}, 3,5-\mathrm{H}_{\text {arom }}\right)$, $7.35-7.42 \mathrm{~m}\left(2 \mathrm{H}, 2,6-\mathrm{H}_{\text {arom }}\right), 8.32 \mathrm{~s}(1 \mathrm{H}, \mathrm{N} \underline{\mathrm{H}}-\mathrm{Ph}) .{ }^{13} \mathrm{C}$ NMR spectrum (DMSO- $\left.d_{6}\right), \delta$, ppm: $28.24(\mathrm{Ad}), 33.95$ (Ad), 37.12 (2C, Ad), 39.71 (3C, Ad), 40.67 (6C, Ad), $51.31\left(2 \mathrm{C}, \mathrm{CH}_{2}\right), 115.47 \mathrm{~d}\left(2 \mathrm{C}, 3,5-\mathrm{C}_{\text {arom }}, J 22.0 \mathrm{~Hz}\right)$, $119.54 \mathrm{~d}\left(2 \mathrm{C}, 2,6-\mathrm{C}_{\text {arom }}, J 7.5 \mathrm{~Hz}\right), 137.51\left(1-\mathrm{C}_{\text {arom }}\right)$, $155.97[\mathrm{NH}-\mathrm{C}(\mathrm{O})-\mathrm{NH}], 158.10\left(4-\mathrm{C}_{\text {arom }}\right) .{ }^{19} \mathrm{~F}$ NMR spectrum (DMSO- $\left.d_{6}\right), \delta$, ppm: -122.87 . Mass spectrum, $m / z\left(I_{\text {rel }}, \%\right): 303(53.8)[M+\mathrm{H}]^{+}, 344(100.0)[M+$ $\mathrm{CAN}+\mathrm{H}]^{+}$. Found, \%: C 71.52; H 7.66; F 6.26; N 9.24. $\mathrm{C}_{18} \mathrm{H}_{23} \mathrm{FN}_{2} \mathrm{O}$. Calculated, \%: C 71.50; H 7.67; F 6.28; N 9.26. M 302.39.

1-(Adamantan-1-ylmethyl)-3-(2,4-difluorophenyl)urea (5c) was prepared similarly to compound 
5a, from $0.25 \mathrm{~g}$ of isocyanate $\mathbf{3}$ and $0.168 \mathrm{~g}$ of 2,4-difluoroaniline. Yield $0.310 \mathrm{~g}(74 \%), \mathrm{mp} 167-168^{\circ} \mathrm{C} .{ }^{1} \mathrm{H}$ NMR spectrum (DMSO- $\left.d_{6}\right), \delta$, ppm: $1.43 \mathrm{~d}(1 \mathrm{H}, \mathrm{Ad}$, $J 2.9 \mathrm{~Hz}), 1.48 \mathrm{~d}(7 \mathrm{H}, \mathrm{Ad}, J 2.9 \mathrm{~Hz}), 1.66 \mathrm{q}(4 \mathrm{H}, \mathrm{Ad}$, $\left.J_{1} 12.9, J_{2} 12.1 \mathrm{~Hz}\right), 1.96$ br.s $(3 \mathrm{H}, \mathrm{Ad}), 2.82 \mathrm{~d}(2 \mathrm{H}$, $\left.\mathrm{CH}_{2}, J 5.8 \mathrm{~Hz}\right), 6.50 \mathrm{q}\left(1 \mathrm{H}, \mathrm{N} \underline{\mathrm{H}}-\mathrm{CH}_{2}-\mathrm{Ad}, J_{1} 6.2, J_{2}\right.$ $\left.6.2, J_{3} 6.0 \mathrm{~Hz}\right), 6.92-6.98 \mathrm{~m}\left(1 \mathrm{H}, 3-\mathrm{H}_{\text {arom }}\right), 7.18$ d.d.d $\left(1 \mathrm{H}, 5-\mathrm{H}_{\text {arom }}, J_{1} 11.7, J_{2} 8.9, J_{3} 2.9 \mathrm{~Hz}\right), 7.20$ t.d $(1 \mathrm{H}$, 6- $\left.\mathrm{H}_{\text {arom }}, J_{1} 9.3, J_{2} 9.3, J_{3} 6.2 \mathrm{~Hz}\right), 8.19 \mathrm{~s}(1 \mathrm{H}, \mathrm{N} \underline{\mathrm{H}}-\mathrm{Ph})$. ${ }^{19} \mathrm{~F}$ NMR spectrum (DMSO- $\left.d_{6}\right), \delta$, ppm: -126.48 (4-F), -120.08 (2-F). Mass spectrum, $m / z\left(I_{\text {rel }}, \%\right): 355$ (26.8) $[M+\mathrm{Cl}]^{-}$. Found, \%: C 67.42; H 6.95; F 11.82; N 8.70. $\mathrm{C}_{18} \mathrm{H}_{22} \mathrm{~F}_{2} \mathrm{~N}_{2} \mathrm{O}$. Calculated, \%: C 67.48; H 6.92; F 11.86; N 8.74. M 320.38.

1-(Adamantan-1-ylmethyl)-3-(2,6-difluorophenyl)urea (5d) was prepared similarly to compound 5a, from $0.25 \mathrm{~g}$ of isocyanate $\mathbf{3}$ and $0.168 \mathrm{~g}$ of 2,6-difluoroaniline. Yield $0.042 \mathrm{~g}(10 \%), \mathrm{mp} 182-183^{\circ} \mathrm{C} .{ }^{1} \mathrm{H}$ NMR spectrum (DMSO- $\left.d_{6}\right), \delta$, ppm: $1.43 \mathrm{~d}(1 \mathrm{H}, \mathrm{Ad}, J$ $2.9 \mathrm{~Hz}), 1.48 \mathrm{~d}(7 \mathrm{H}, \mathrm{Ad}, J 2.8 \mathrm{~Hz}), 1.66 \mathrm{q}\left(4 \mathrm{H}, \mathrm{Ad}, J_{1}\right.$ $\left.12.3, J_{2} 12.3 \mathrm{~Hz}\right), 1.96$ br.s (3H, Ad), $2.80 \mathrm{~d}\left(2 \mathrm{H}, \mathrm{CH}_{2}, J\right.$ $5.9 \mathrm{~Hz}), 6.26 \mathrm{~s}\left(1 \mathrm{H}, \mathrm{N} \underline{\mathrm{H}}-\mathrm{CH}_{2}-\mathrm{Ad}\right), 7.06 \mathrm{t}\left(2 \mathrm{H}, 3,5-\mathrm{H}_{\text {arom }}\right.$, $J 8.0 \mathrm{~Hz}), 7.22$ t.t $\left(1 \mathrm{H}, 4-\mathrm{H}_{\text {arom }}, J_{1} 8.3, J_{2} 8.3, J_{3} 6.2 \mathrm{~Hz}\right)$, $7.75 \mathrm{~s}(1 \mathrm{H}, \mathrm{NH}-\mathrm{Ph}) .{ }^{19} \mathrm{~F}$ NMR spectrum (DMSO- $d_{6}$ ), $\delta$, ppm: $-119.08(2 \mathrm{~F}, 2,6-\mathrm{F})$. Mass spectrum, $\mathrm{m} / \mathrm{z}$ $\left(I_{\text {rel }}, \%\right): 355$ (9.9) $[M+\mathrm{Cl}]^{-}$. Found, \%: C 67.45; H 6.90; F 11.88; N 8.76. $\mathrm{C}_{18} \mathrm{H}_{22} \mathrm{~F}_{2} \mathrm{~N}_{2} \mathrm{O}$. Calculated, \%: C 67.48; H 6.92; F 11.86; N 8.74. M 320.38.

1-(Adamantan-1-ylmethyl)-3-(3,4-difluorophenyl)urea (5e) was prepared similarly to compound 5a, from $0.25 \mathrm{~g}$ of isocyanate $\mathbf{3}$ and $0.168 \mathrm{~g}$ of 3,4-difluoroaniline. Yield $0.291 \mathrm{~g}(70 \%), \mathrm{mp} 142-143^{\circ} \mathrm{C} .{ }^{1} \mathrm{H}$ NMR spectrum (DMSO- $\left.d_{6}\right), \delta$, ppm: $1.43 \mathrm{~d}(1 \mathrm{H}, \mathrm{Ad}, J$ $2.9 \mathrm{~Hz}), 1.47 \mathrm{~d}(6 \mathrm{H}, \mathrm{Ad}, J 2.9 \mathrm{~Hz}), 1.65 \mathrm{q}\left(4 \mathrm{H}, \mathrm{Ad}, J_{1}\right.$ $\left.12.1, J_{2} 12.1 \mathrm{~Hz}\right), 1.95$ br.s ( $\left.3 \mathrm{H}, \mathrm{Ad}\right), 2.81 \mathrm{~d}\left(2 \mathrm{H}, \mathrm{CH}_{2}\right.$, $J 6.0 \mathrm{~Hz}), 6.13 \mathrm{t}\left(1 \mathrm{H}, \mathrm{N} \underline{\mathrm{H}}-\mathrm{CH}_{2}-\mathrm{Ad}, J_{1} 6.1, J_{2} 6.1 \mathrm{~Hz}\right)$, 7.00 d.d.d (1H, $\left.5-\mathrm{H}_{\text {arom }}, J_{1} 9.3, J_{2} 4.2, J_{3} 2.0 \mathrm{~Hz}\right), 7.23$ d.t $\left(1 \mathrm{H}, 2-\mathrm{H}_{\text {arom }}, J_{1} 10.5, J_{2} 9.1, J_{3} 9.1 \mathrm{~Hz}\right), 7.62$ d.d.d $(1 \mathrm{H}$, $\left.6-\mathrm{H}_{\text {arom }}, J_{1} 13.8, J_{2} 7.5, J_{3} 2.7 \mathrm{~Hz}\right), 8.57 \mathrm{~s}(1 \mathrm{H}, \mathrm{N} \underline{\mathrm{H}}-\mathrm{Ph})$. ${ }^{19} \mathrm{~F}$ NMR spectrum (DMSO- $\left.d_{6}\right), \delta$, ppm: -148.57 (4-F), -137.77 (3-F). Mass spectrum, $m / z$ ( $\left.I_{\text {rel }}, \%\right): 319$ (2.3) $[M-\mathrm{H}]^{-}, 355(100.0)[M+\mathrm{Cl}]^{-}$. Found, \%: C 67.44; H 6.93; F 11.83; N 8.72. $\mathrm{C}_{18} \mathrm{H}_{22} \mathrm{~F}_{2} \mathrm{~N}_{2} \mathrm{O}$. Calculated, \%: C 67.48; H 6.92; F 11.86; N 8.74. M 320.38.

1-(Adamantan-1-ylmethyl)-3-(2,5-difluorophenyl)urea (5f) was prepared similarly to compound 5a, from $0.25 \mathrm{~g}$ of isocyanate $\mathbf{3}$ and $0.168 \mathrm{~g}$ of 2,5 -di- fluoroaniline. Yield $0.341 \mathrm{~g}(82 \%), \mathrm{mp} 92-93^{\circ} \mathrm{C} .{ }^{1} \mathrm{H}$ NMR spectrum (DMSO- $\left.d_{6}\right), \delta$, ppm: $1.43 \mathrm{~d}(1 \mathrm{H}, \mathrm{Ad}$, $J 2.9 \mathrm{~Hz}), 1.48 \mathrm{~d}(6 \mathrm{H}, \mathrm{Ad}, J 2.9 \mathrm{~Hz}), 1.65 \mathrm{q}(4 \mathrm{H}, \mathrm{Ad}$, $\left.J_{1} 11.3, J_{2} 11.3 \mathrm{~Hz}\right), 1.95$ br.s $(3 \mathrm{H}, \mathrm{Ad}), 2.83 \mathrm{~d}(2 \mathrm{H}$, $\left.\mathrm{CH}_{2}, J 5.8 \mathrm{~Hz}\right), 5.68 \mathrm{~s}\left(1 \mathrm{H}, \mathrm{N} \underline{\mathrm{H}}-\mathrm{CH}_{2}-\mathrm{Ad}\right), 6.64-6.72 \mathrm{~m}$ (4- $\left.\mathrm{H}_{\text {arom }}\right), 6.85$ t.t $\left(1 \mathrm{H}, 3-\mathrm{H}_{\text {arom }}, J_{1} 8.7, J_{2} 8.7, J_{3} 3.4\right.$, $\left.J_{4} 3.4 \mathrm{~Hz}\right), 7.29$ d.d.d $\left(1 \mathrm{H}, 6-\mathrm{H}_{\text {arom }}, J_{1} 11.1, J_{2} 9.0\right.$, $\left.J_{3} 5.2 \mathrm{~Hz}\right), 8.46 \mathrm{~s}(1 \mathrm{H}, \mathrm{N} \underline{\mathrm{H}}-\mathrm{Ph}) .{ }^{19} \mathrm{~F}$ NMR spectrum (DMSO- $d_{6}$ ), $\delta$, ppm: -135.09 (5-F), -116.70 (2-F). Mass spectrum, $m / z\left(I_{\text {rel }}, \%\right): 319(2.0)[M-\mathrm{H}]^{-}, 355$ (31.0) $[M+\mathrm{Cl}]^{-}$. Found, \%: C 67.49; H 6.96; F 11.81; $\mathrm{N}$ 8.77. $\mathrm{C}_{18} \mathrm{H}_{22} \mathrm{~F}_{2} \mathrm{~N}_{2} \mathrm{O}$. Calculated, \%: C 67.48; H 6.92; F 11.86; N 8.74. $M 320.38$.

1-(Adamantan-1-ylmethyl)-3-(3,5-difluorophenyl)urea (5g) was prepared similarly to compound 5a, from $0.25 \mathrm{~g}$ of isocyanate 3 and $0.168 \mathrm{~g}$ of 3,5-difluoroaniline. Yield $0.291 \mathrm{~g}(70 \%), \mathrm{mp} 124-125^{\circ} \mathrm{C} .{ }^{1} \mathrm{H}$ NMR spectrum (DMSO- $\left.d_{6}\right), \delta$, ppm: $1.43 \mathrm{~d}(1 \mathrm{H}, \mathrm{Ad}, J$ $2.9 \mathrm{~Hz}), 1.47 \mathrm{~d}(6 \mathrm{H}, \mathrm{Ad}, J 2.9 \mathrm{~Hz}), 1.65 \mathrm{q}\left(4 \mathrm{H}, \mathrm{Ad}, J_{1}\right.$ 9.3, $\left.J_{2} 9.0 \mathrm{~Hz}\right), 1.94$ br.s (3H, Ad), $2.82 \mathrm{~d}\left(2 \mathrm{H}, \mathrm{CH}_{2}, J\right.$ $5.8 \mathrm{~Hz}), 6.24 \mathrm{~s}\left(1 \mathrm{H}, \mathrm{N} \underline{\mathrm{H}}-\mathrm{CH}_{2}-\mathrm{Ad}\right), 6.79$ d.t $\left(1 \mathrm{H}, 4-\mathrm{H}_{\text {arom }}\right.$, $\left.J_{1} 9.3, J_{2} 2.4, J_{3} 2.4 \mathrm{~Hz}\right), 7.10$ d.d $\left(1 \mathrm{H}, 2-\mathrm{H}_{\text {arom }}, J_{1} 10.2\right.$, $\left.J_{2} 2.3 \mathrm{~Hz}\right), 7.20$ d.d $\left(1 \mathrm{H}, 6-\mathrm{H}_{\text {arom }}, J_{1} 7.6, J_{2} 2.3 \mathrm{~Hz}\right)$, $7.96 \mathrm{~s}(1 \mathrm{H}, \mathrm{N} \underline{\mathrm{H}}-\mathrm{Ph}) .{ }^{19} \mathrm{~F}$ NMR spectrum (DMSO- $\left.d_{6}\right)$, $\delta$, ppm: $-110.01(2 \mathrm{~F}, 3,5-\mathrm{F})$. Mass spectrum, $\mathrm{m} / z$ $\left(I_{\text {rel }}, \%\right): 319(4.0)[M-\mathrm{H}]^{-}, 355$ (100.0) $[M+\mathrm{Cl}]^{-}$. Found, \%: C 67.44; H 6.90; F 11.89; N 8.71. $\mathrm{C}_{18} \mathrm{H}_{22} \mathrm{~F}_{2} \mathrm{~N}_{2} \mathrm{O}$. Calculated, \%: C 67.48; H 6.92; F 11.86; N 8.74. $M 320.38$.

1-(Adamantan-1-ylmethyl)-3-(3-chlorophenyl)urea (5h) was prepared similarly to compound 5a, from $0.25 \mathrm{~g}$ of isocyanate 3 and $0.165 \mathrm{~g}$ of 3-chloroaniline. Yield $0.382 \mathrm{~g}(92 \%), \mathrm{mp} 163-164^{\circ} \mathrm{C} .{ }^{1} \mathrm{H}$ NMR spectrum (DMSO- $\left.d_{6}\right), \delta$, ppm: $1.45 \mathrm{~d}(6 \mathrm{H}, \mathrm{Ad}, J 2.2 \mathrm{~Hz}), 1.64 \mathrm{q}$ $\left(6 \mathrm{H}, \mathrm{Ad}, J_{1} 11.7, J_{2} 12.0 \mathrm{~Hz}\right), 1.94 \mathrm{~s}(3 \mathrm{H}, \mathrm{Ad}), 2.80 \mathrm{~d}$ $\left(2 \mathrm{H}, \mathrm{CH}_{2}, J 6.1 \mathrm{~Hz}\right), 6.15 \mathrm{~s}\left(1 \mathrm{H}, \mathrm{N} \underline{\mathrm{H}}-\mathrm{CH}_{2}-\mathrm{Ad}\right), 6.91 \mathrm{~d}$ $\left(1 \mathrm{H}, 4-\mathrm{H}_{\text {arom }}, J 7.9 \mathrm{~Hz}\right), 7.19 \mathrm{~d}\left(1 \mathrm{H}, 5-\mathrm{H}_{\text {arom }}, J_{1} 8.1 \mathrm{~Hz}\right)$, $7.22 \mathrm{t}\left(1 \mathrm{H}, 6-\mathrm{H}_{\text {arom }}, J_{1} 8.0, J_{2} 8.1 \mathrm{~Hz}\right), 7.68 \mathrm{~d}(1 \mathrm{H}$, $\left.2-\mathrm{H}_{\text {arom }}, J 6.0 \mathrm{~Hz}\right), 8.56 \mathrm{~s}(1 \mathrm{H}, \mathrm{N} \underline{\mathrm{H}}-\mathrm{Ph})$. Mass spectrum, $m / z\left(I_{\text {rel }}, \%\right): 318(11.5)[M]^{+}, 127(100.0)\left[\mathrm{C}_{6} \mathrm{H}_{4} \mathrm{ClNH}\right]^{+}$, 135 (100.0) [Ad] $]^{+}$. Found, \%: C 67.85; H 7.24; Cl 11.10; $\mathrm{N}$ 8.76. $\mathrm{C}_{18} \mathrm{H}_{23} \mathrm{ClN}_{2} \mathrm{O}$. Calculated, \%: C 67.81; H 7.27; Cl 11.12; N 8.79. M 318.84.

1-(Adamantan-1-ylmethyl)-3-(2-chloro-4-fluorophenyl)urea (5i) was prepared similarly to compound 5a, from $0.25 \mathrm{~g}$ of isocyanate 3 and $0.189 \mathrm{~g}$ of 2-chloro4-fluoroaniline. Yield $0.315 \mathrm{~g}(72 \%), \mathrm{mp} 161-162^{\circ} \mathrm{C}$. 
${ }^{1} \mathrm{H}$ NMR spectrum (DMSO- $\left.d_{6}\right), \delta$, ppm: $1.43 \mathrm{~d}(1 \mathrm{H}$, $\mathrm{Ad}, J 2.8 \mathrm{~Hz}), 1.49 \mathrm{~d}(6 \mathrm{H}, \mathrm{Ad}, J 2.9 \mathrm{~Hz}), 1.64 \mathrm{q}(4 \mathrm{H}$, $\left.\mathrm{Ad}, J_{1} 11.3, J_{2} 11.0 \mathrm{~Hz}\right), 1.93$ br.s $(3 \mathrm{H}, \mathrm{Ad}), 2.83 \mathrm{~d}(2 \mathrm{H}$, $\left.\mathrm{CH}_{2}, J 5.7 \mathrm{~Hz}\right), 6.81 \mathrm{~d}\left(1 \mathrm{H}, \mathrm{N} \underline{\mathrm{H}}-\mathrm{CH}_{2}-\mathrm{Ad}, J 5.9 \mathrm{~Hz}\right)$, $7.10-7.22 \mathrm{~m}\left(1 \mathrm{H}, 5-\mathrm{H}_{\text {arom }}\right), 7.36$ t.d $\left(1 \mathrm{H}, 3-\mathrm{H}_{\text {arom }}, J_{1}\right.$ 8.7, $\left.J_{2} 8.5, J_{3} 3.0 \mathrm{~Hz}\right), 7.62-7.67 \mathrm{~m}\left(1 \mathrm{H}, 6-\mathrm{H}_{\text {arom }}\right)$, 8.14 d.d.d $\left(1 \mathrm{H}, \mathrm{N} \underline{\mathrm{H}}-\mathrm{Ph}, J_{1} 9.4, J_{2} 5.9, J_{3} 3.7 \mathrm{~Hz}\right) .{ }^{19} \mathrm{~F}$ NMR spectrum (DMSO- $\left.d_{6}\right), \delta$, ppm: -120.52 . Mass spectrum, $m / z\left(I_{\text {rel }}, \%\right): 338(1.2)[M+\mathrm{H}]^{+}, 355(1.1)$ $\left[M+\mathrm{H}_{2} \mathrm{O}\right]^{+}, 379(1.0)[M+\mathrm{CAN}+\mathrm{H}]^{+}, 420(42.1)$ $[M+2 \mathrm{ACN}+\mathrm{H}]^{+}$. Found, \%: C 64.17; H 6.56; Cl 10.55; F 5.62; N 8.34. $\mathrm{C}_{18} \mathrm{H}_{22} \mathrm{ClFN}_{2} \mathrm{O}$. Calculated, \%: C 64.18; H 6.58; Cl 10.53; F 5.64; N 8.32. M 336.83.

1-(Adamantan-1-ylmethyl)-3-(3-chloro-4-fluorophenyl)urea (5j) was prepared similarly to compound 5a, from $0.25 \mathrm{~g}$ of isocyanate 3 and $0.189 \mathrm{~g}$ of 3-chloro4-fluoroaniline. Yield $0.320 \mathrm{~g}(73 \%), \mathrm{mp} 115-116^{\circ} \mathrm{C}$. ${ }^{1} \mathrm{H}$ NMR spectrum (DMSO- $\left.d_{6}\right), \delta$, ppm: $1.43 \mathrm{~d}(1 \mathrm{H}, \mathrm{Ad}$, $J 2.9 \mathrm{~Hz}), 1.48 \mathrm{~d}(6 \mathrm{H}, \mathrm{Ad}, J 2.9 \mathrm{~Hz}), 1.66 \mathrm{q}\left(4 \mathrm{H}, \mathrm{Ad}, J_{1}\right.$ $\left.11.6, J_{2} 11.6 \mathrm{~Hz}\right), 1.93$ br.s $(3 \mathrm{H}, \mathrm{Ad}), 2.83 \mathrm{~d}\left(2 \mathrm{H}, \mathrm{CH}_{2}\right.$, $J 5.7 \mathrm{~Hz}), 6.62 \mathrm{t}\left(1 \mathrm{H}, \mathrm{N} \underline{\mathrm{H}}-\mathrm{CH}_{2}-\mathrm{Ad}, J_{1} 6.2, J_{2} 6.2 \mathrm{~Hz}\right)$, 7.04-7.10 m (1H, 5- $\left.\mathrm{H}_{\text {arom }}\right), 7.17-7.22 \mathrm{~m}\left(1 \mathrm{H}, 6-\mathrm{H}_{\text {arom }}\right)$, $8.10-8.15 \mathrm{~m}\left(1 \mathrm{H}, 2-\mathrm{H}_{\text {arom }}\right), 8.40 \mathrm{~s}(1 \mathrm{H}, \mathrm{N} \underline{\mathrm{H}}-\mathrm{Ph}) .{ }^{19} \mathrm{~F}$ NMR spectrum (DMSO- $\left.d_{6}\right), \delta$, ppm: -133.42 . Mass spectrum, $m / z\left(I_{\text {rel }}, \%\right): 420(100.0)[M+2 \mathrm{ACN}+\mathrm{H}]^{+}$. Found, \%: C 64.19; H 6.59; Cl 10.56; F 5.66; N 8.30. $\mathrm{C}_{18} \mathrm{H}_{22} \mathrm{ClFN}_{2} \mathrm{O}$. Calculated, \%: C 64.18; H 6.58; Cl 10.53; F 5.64; N 8.32. M 336.83.

1-(Adamantan-1-ylmethyl)-3-(3-chloro-2-fluorophenyl)urea (5k) was prepared similarly to compound 5a, from $0.25 \mathrm{~g}$ of isocyanate $\mathbf{3}$ and $0.189 \mathrm{~g}$ 3-chloro2-fluoroaniline. Yield $0.375 \mathrm{~g}(85 \%), \mathrm{mp} 83-84^{\circ} \mathrm{C} .{ }^{1} \mathrm{H}$ NMR spectrum (DMSO- $\left.d_{6}\right), \delta$, ppm: $1.43 \mathrm{~d}(1 \mathrm{H}, \mathrm{Ad}, J$ $2.9 \mathrm{~Hz}), 1.47 \mathrm{~d}(6 \mathrm{H}, \mathrm{Ad}, J 2.9 \mathrm{~Hz}), 1.64 \mathrm{q}\left(4 \mathrm{H}, \mathrm{Ad}, J_{1}\right.$ $\left.11.1, J_{2} 11.2 \mathrm{~Hz}\right), 1.94$ br.s (3H, Ad), $2.81 \mathrm{~d}\left(2 \mathrm{H}, \mathrm{CH}_{2}, J\right.$ $5.2 \mathrm{~Hz}), 6.13 \mathrm{~s}\left(1 \mathrm{H}, \mathrm{N} \underline{\mathrm{H}}-\mathrm{CH}_{2}-\mathrm{Ad}\right), 7.24 \mathrm{t}\left(1 \mathrm{H}, 5-\mathrm{H}_{\text {arom }}\right.$, $\left.J_{1} 9.0, J_{2} 9.0 \mathrm{~Hz}\right), 7.32 \mathrm{~d}\left(1 \mathrm{H}, 4-\mathrm{H}_{\text {arom }}, J 7.9 \mathrm{~Hz}\right), 7.73-$ $7.80 \mathrm{~m}\left(1 \mathrm{H}, 6-\mathrm{H}_{\text {arom }}, J_{1} 12.7, J_{2} 6.8, J_{3} 2.1 \mathrm{~Hz}\right), 7.96 \mathrm{~s}$ $(1 \mathrm{H}, \mathrm{N} \underline{\mathrm{H}}-\mathrm{Ph}) .{ }^{19} \mathrm{~F}$ NMR spectrum (DMSO- $\left.d_{6}\right), \delta, \mathrm{ppm}$ : -126.57 . Mass spectrum, $m / z\left(I_{\text {rel }}, \%\right): 318$ (7.4) $[M-$ $\left.\mathrm{H}_{2} \mathrm{O}-\mathrm{H}\right]^{-}, 372(2.1)[M+\mathrm{Cl}]^{-}$. Found, \%: C 64.13; $\mathrm{H}$ 6.53; $\mathrm{Cl} 10.58 ; \mathrm{F}$ 5.69; N 8.34. $\mathrm{C}_{18} \mathrm{H}_{22} \mathrm{ClFN}_{2} \mathrm{O}$. Calculated, \%: C 64.18; H 6.58; Cl 10.53; F 5.64; N 8.32. M 336.83.

1-(Adamantan-1-ylmethyl)-3-(4-chloro-3-fluorophenyl)urea (5l) was prepared similarly to compound 5a, from $0.25 \mathrm{~g}$ of isocyanate $\mathbf{3}$ and $0.189 \mathrm{~g}$ OF 4-chloro- 3-fluoroaniline. ${ }^{1} \mathrm{H}$ NMR spectrum (DMSO- $\left.d_{6}\right), \delta$, ppm: $1.43 \mathrm{~d}(1 \mathrm{H}, \mathrm{Ad}, J 2.9 \mathrm{~Hz}), 1.47 \mathrm{~d}(6 \mathrm{H}, \mathrm{Ad}, J 2.9 \mathrm{~Hz})$, $1.64 \mathrm{q}\left(4 \mathrm{H}, \mathrm{Ad}, J_{1} 12.4, J_{2} 10.6 \mathrm{~Hz}\right), 1.94$ br.s $(3 \mathrm{H}, \mathrm{Ad})$, $2.81 \mathrm{~d}\left(2 \mathrm{H}, \mathrm{CH}_{2}, J 5.8 \mathrm{~Hz}\right), 6.21 \mathrm{t}\left(1 \mathrm{H}, \mathrm{N} \underline{\mathrm{H}}-\mathrm{CH}_{2}-\mathrm{Ad}\right.$, $\left.J_{1} 6.1, J_{2} 6.1 \mathrm{~Hz}\right), 7.20$ d.d.d $\left(1 \mathrm{H}, 5-\mathrm{H}_{\text {arom }}, J_{1} 8.8, J_{2}\right.$ $\left.2.5, J_{3} 1.0 \mathrm{~Hz}\right), 7.37 \mathrm{t}\left(1 \mathrm{H}, 2-\mathrm{H}_{\text {arom }}, J_{1} 8.7, J_{2} 8.7 \mathrm{~Hz}\right)$, 7.63 t.d $\left(1 \mathrm{H}, 6-\mathrm{H}_{\text {arom }}, J_{1} 12.4, J_{2} 12.1, J_{3} 2.4 \mathrm{~Hz}\right), 7.96 \mathrm{~s}$ $(1 \mathrm{H}, \mathrm{N} \underline{\mathrm{H}}-\mathrm{Ph}) .{ }^{19} \mathrm{~F}$ NMR spectrum (DMSO- $\left.d_{6}\right), \delta$, ppm: -115.37 . Mass spectrum, $m / z\left(I_{\text {rel }}, \%\right): 318$ (9.0) [M$\left.\mathrm{H}_{2} \mathrm{O}-\mathrm{H}\right]^{-}, 372(2.0)[M+\mathrm{Cl}]^{-}$. Found, \%: C 64.14; $\mathrm{H}$ 6.55; $\mathrm{Cl} 10.50 ; \mathrm{F}$ 5.69; N 8.35. $\mathrm{C}_{18} \mathrm{H}_{22} \mathrm{ClFN}_{2} \mathrm{O}$. Calculated, \%: C 64.18; H 6.58; Cl 10.53; F 5.64; N 8.32. M 336.83.

1-(Adamantan-1-ylmethyl)-3-(4-chloro-2-fluorophenyl)urea $(\mathbf{5 m})$ was prepared similarly to compound 5a, from $0.25 \mathrm{~g}$ of isocyanate $\mathbf{3}$ and $0.189 \mathrm{~g}$ of 4-chloro2-fluoroaniline. Yield $0.335 \mathrm{~g}(76 \%), \mathrm{mp} 109-110^{\circ} \mathrm{C}$. ${ }^{1} \mathrm{H}$ NMR spectrum (DMSO- $\left.d_{6}\right), \delta$, ppm: $1.43 \mathrm{~d}(1 \mathrm{H}, \mathrm{Ad}$, $J 2.9 \mathrm{~Hz}), 1.47 \mathrm{~d}(6 \mathrm{H}, \mathrm{Ad}, J 2.9 \mathrm{~Hz}), 1.65 \mathrm{q}\left(4 \mathrm{H}, \mathrm{Ad}, J_{1}\right.$ $\left.12.3, J_{2} 12.2 \mathrm{~Hz}\right), 1.94$ br.s $(3 \mathrm{H}, \mathrm{Ad}), 2.82 \mathrm{~d}\left(2 \mathrm{H}, \mathrm{CH}_{2}\right.$, $J 5.7 \mathrm{~Hz}), 6.59 \mathrm{t}\left(1 \mathrm{H}, \mathrm{N} \underline{\mathrm{H}}-\mathrm{CH}_{2}-\mathrm{Ad}, J_{1} 6.0, J_{2} 6.0 \mathrm{~Hz}\right)$, $7.14 \mathrm{~d}\left(1 \mathrm{H}, 5-\mathrm{H}_{\text {arom }}, J 8.9 \mathrm{~Hz}\right), 7.34 \mathrm{~d} . \mathrm{d}\left(1 \mathrm{H}, 6-\mathrm{H}_{\text {arom }}, J_{1}\right.$ $\left.2.4, J_{2} 11.3 \mathrm{~Hz}\right), 8.18 \mathrm{t}\left(1 \mathrm{H}, 3-\mathrm{H}_{\text {arom }}, J 8.2 \mathrm{~Hz}\right), 8.34 \mathrm{~d}$ $(1 \mathrm{H}, \mathrm{N} \underline{\mathrm{H}}-\mathrm{Ph}, J 2.7 \mathrm{~Hz}) .{ }^{19} \mathrm{~F}$ NMR spectrum (DMSO- $\left.d_{6}\right)$, $\delta$, ppm: -128.09 . Mass spectrum, $m / z\left(I_{\text {rel }}, \%\right): 336$ (3.8) $[M-\mathrm{H}]^{-}, 372(15.2)[M+\mathrm{Cl}]^{-}$. Found, \%: C 64.15; $\mathrm{H}$ 6.55; Cl 10.57; F 5.60; N 8.37. $\mathrm{C}_{18} \mathrm{H}_{22} \mathrm{ClFN}_{2} \mathrm{O}$. Calculated, \%: C 64.18; H 6.58; Cl 10.53; F 5.64; N 8.32. M336.83.

1-(Adamantan-1-ylmethyl)-3-(6-chloro-2-fluorophenyl)urea (5n) was prepared similarly to compound 5a, from $0.25 \mathrm{~g}$ of isocyanate 3 and $0.189 \mathrm{~g}$ of 6-chloro2-fluoroaniline. Yield $0.348 \mathrm{~g}(79 \%)$, mp $113-114^{\circ} \mathrm{C}$. ${ }^{1} \mathrm{H}$ NMR spectrum (DMSO- $\left.d_{6}\right), \delta$, ppm: $1.43 \mathrm{~d}(1 \mathrm{H}$, Ad, $J 2.5 \mathrm{~Hz}), 1.48 \mathrm{~d}(6 \mathrm{H}, \mathrm{Ad}, J 2.9 \mathrm{~Hz}), 1.65 \mathrm{q}(4 \mathrm{H}$, Ad, $\left.J_{1} 11.4, J_{2} 11.4 \mathrm{~Hz}\right), 1.95$ br.s ( $\left.3 \mathrm{H}, \mathrm{Ad}\right), 2.83 \mathrm{~d}(2 \mathrm{H}$, $\left.\mathrm{CH}_{2}, J 5.8 \mathrm{~Hz}\right), 6.67 \mathrm{t}\left(1 \mathrm{H}, \mathrm{N} \underline{\mathrm{H}}-\mathrm{CH}_{2}-\mathrm{Ad}, J_{1} 6.0, J_{2}\right.$ $6.0 \mathrm{~Hz}), 7.09$ d.d.d $\left(1 \mathrm{H}, 3-\mathrm{H}_{\text {arom }}, J_{1} 8.7, J_{2} 4.2, J_{3}\right.$ $2.7 \mathrm{~Hz}), 7.20$ d.d $\left(1 \mathrm{H}, 4-\mathrm{H}_{\text {arom }}, J_{1} 11.3, J_{2} 8.7 \mathrm{~Hz}\right)$, 7.30 d.d $\left(1 \mathrm{H}, 5-\mathrm{H}_{\text {arom }}, J_{1} 11.1, J_{2} 8.8 \mathrm{~Hz}\right), 7.96 \mathrm{~s}(1 \mathrm{H}$, $\mathrm{NH}-\mathrm{Ph}) .{ }^{19} \mathrm{~F}$ NMR spectrum (DMSO- $\left.d_{6}\right), \delta$, ppm: -133.10. Mass spectrum, $m / z\left(I_{\text {rel }}, \%\right): 336(4.9)[M-$ $\mathrm{H}]^{-}, 372(11.8)[M+\mathrm{Cl}]^{-}$. Found, \%: C 64.17; H 6.56; $\mathrm{Cl} 10.52 ; \mathrm{F} 5.69 ; \mathrm{N}$ 8.36. $\mathrm{C}_{18} \mathrm{H}_{22} \mathrm{ClFN}_{2} \mathrm{O}$. Calculated, \%: C 64.18; H 6.58; Cl 10.53; F 5.64; N 8.32. M 336.83.

1-(Adamantan-1-ylmethyl)-3-[3-(trifluoromethyl)phenyl]urea (5o) was prepared similarly to 
compound $\mathbf{5 a}$, from $0.25 \mathrm{~g}$ of isocyanate $\mathbf{3}$ and $0.211 \mathrm{~g}$ of 3-(trifluoromethyl)aniline. Yield $0.327 \mathrm{~g}$ (71\%), mp $153-155^{\circ} \mathrm{C} .{ }^{1} \mathrm{H}$ NMR spectrum (DMSO- $d_{6}$ ), $\delta$, ppm: $1.43 \mathrm{~s}(1 \mathrm{H}, \mathrm{Ad}), 1.48 \mathrm{~s}(6 \mathrm{H}, \mathrm{Ad}), 1.64 \mathrm{q}(4 \mathrm{H}$, Ad, $\left.J_{1} 12.7, J_{2} 11.3 \mathrm{~Hz}\right), 1.95$ br.s $(3 \mathrm{H}, \mathrm{Ad}), 2.83 \mathrm{~d}$ $\left(2 \mathrm{H}, \mathrm{CH}_{2}, J 5.8 \mathrm{~Hz}\right), 6.19 \mathrm{~s}\left(1 \mathrm{H}, \mathrm{N} \underline{\mathrm{H}}-\mathrm{CH}_{2}-\mathrm{Ad}\right), 7.20 \mathrm{~d}$ $\left(1 \mathrm{H}, 4-\mathrm{H}_{\text {arom }}, J 7.5 \mathrm{~Hz}\right), 7.33 \mathrm{~d}\left(1 \mathrm{H}, 5-\mathrm{H}_{\text {arom }}, J 7.8 \mathrm{~Hz}\right)$, $7.53 \mathrm{t}\left(1 \mathrm{H}, 6-\mathrm{H}_{\text {arom }}, J_{1} 8.0, J_{2} 8.0 \mathrm{~Hz}\right), 7.62 \mathrm{~d}(1 \mathrm{H}$, $\left.2-\mathrm{H}_{\text {arom }}, J 8.2 \mathrm{~Hz}\right), 7.98 \mathrm{~s}(1 \mathrm{H}, \mathrm{N} \underline{\mathrm{H}}-\mathrm{Ph}) .{ }^{13} \mathrm{C}$ NMR spectrum (DMSO- $d_{6}$ ), $\delta$, ppm: $28.26(\mathrm{Ad}), 33.98$ (Ad), 37.14 (2C, Ad), 39.71 (3C, Ad), 40.74 (3C, Ad), 51.67 (2C, $\left.\mathrm{CH}_{2}\right), 113.92\left(2-\mathrm{C}_{\text {arom }}\right), 114.98\left(4-\mathrm{C}_{\text {arom }}\right), 117.51\left(\mathrm{CF}_{3}\right)$, $118.87\left(6-\mathrm{C}_{\text {arom }}\right), 122.63\left(5-\mathrm{C}_{\text {arom }}\right), 130.18\left(3-\mathrm{C}_{\text {arom }}\right)$, $140.81\left(1-\mathrm{C}_{\text {arom }}\right), 159.14$ [NH-C(O)-NH]. ${ }^{19} \mathrm{~F}$ NMR spectrum (DMSO- $\left.d_{6}\right), \delta$, ppm: $-61.33\left(3 \mathrm{~F}, \mathrm{CF}_{3}\right)$. Mass spectrum, $m / z\left(I_{\text {rel }}, \%\right): 351(1.0)[M-\mathrm{H}]^{-}, 387(15.0)$ $[M+\mathrm{Cl}]^{-}$. Found, \%: C 64.71; H 6.56; F 16.19, N 7.91. $\mathrm{C}_{19} \mathrm{H}_{23} \mathrm{~F}_{3} \mathrm{~N}_{2} \mathrm{O}$. Calculated, \%: C 64.76; H 6.58; F 16.17; N 7.95. M 352.39.

\section{CONCLUSIONS}

Methods of synthesis of adamantane-containing 1,3-disubstituted ureas with a methylene bridge between the adamantane and urea moieties are developed. The advantages of the developed methods include cheap starting materials and facile protocols of synthesis and isolation and purification of the products. An alternative, safer method of synthesis of 1-(isocyanato)adamantane (3). The synthesized compounds will be studied as human soluble epoxide hydrolase inhibitors.

\section{FUNDING}

The work was financially supported by the Russian Science Foundation (project no. 19-73-10002).

\section{CONFLICT OF INTEREST}

The authors declare no conflict of interest.

\section{REFERENCES}

1. Pitushkin, D.A., Burmistrov, V.V., Kuznetsov, Y.P., Ivankina, O.M., and Butov G.M., Russ. J. Org. Chem., 2020, vol. 56.

https://doi.org/10.1134/S1070428020120027

2. Park, S.J., Kim, Y.G., and Park, H.J., J. Am. Chem. Soc., 2011, vol. 133, p. 10094.

https://doi.org/10.1021/ja1098325

3. Brown, J.R., North, E.J., Hurdle, J.G., Morisseau, C., Scarborough, J.S., Sun, D., Korduláková, J., Scher- man, M.S., Jones, V., Grzegorzewicz, A., Crew, R.M., Jackson, M., McNeil, M.R., and Lee, R.E., Bioorg. Med. Chem., 2011, vol. 19, p. 5585.

https://doi.org/10.1016/j.bmc.2011.07.034

4. Wanka, L., Iqbal, K., and Schreiner, P.R., Chem. Rev., 2013, vol. 113, p. 3516.

https://doi.org/10.1021/cr100264t

5. Dorrance, A.M., Rupp, N., Pollock, D.M., Newman, J.W., Hammock, B.D., and Imig, J.D., J. Cardiovasc. Pharmacol., 2005, vol. 46, p. 842.

https://doi.org/10.1097/01.fjc.0000189600.74157.6d

6. Liu, J.Y., Yang, J., Inceoglu, B., Qiu, H., Ulu, A., Hwang, S.H., Chiamvimonvat, N., and Hammock, B.D., Biochem. Pharm., 2010, vol. 79, p. 880.

https://doi.org/10.1016/j.bcp.2009.10.025

7. Manhiani, M., Quigley, J.E., Knight, S.F., Tasoobshirazi, S., Moore, T., Brands, M.W., Hammock, B.D., and Imig, J.D., Am. J. Physiol. Renal. Physiol., 2009, vol. 297, p. F740.

https://doi.org/10.1152/ajprenal.00098.2009

8. Hwang, S.H., Wecksler, A.T., Zhang, G., Morisseau, C., Nguyen, L.V., Fu, S.H., and Hammock, B.D., Bioorg. Med. Chem. Lett., 2013, vol. 23, p. 3732. https://doi.org/10.1016/j.bmcl.2013.05.011

9. Codony, S., Valverde, E., Leiva, R., Brea, J., Loza, M.I., Morisseau, C., Hammock, B.D., and Vázquez, S., Bioorg. Med. Chem., 2019, vol. 27, p. 115078.

https://doi.org/10.1016/j.bmc.2019.115078

10. Burmistrov, V., Morisseau, C., Harris, T.R., Butov, G., and Hammock, B.D., Bioorg. Chem., 2018, vol. 76, p. 510. https://doi.org/10.1016/j.bioorg.2017.12.024

11. Butov, G.M., Burmistrov, V.V., and Pitushkin, D.A., Russ. J. Org. Chem., 2017, vol. 53, p. 673. https://doi.org/10.1134/s1070428017050050

12. Danilov, D.V., Burmistrov, V.V., and Butov, G.M., Russ. J. Org. Chem., 2020, vol. 56, p. 1004. https://doi.org/10.31857/S0514749220070022

13. Lipinski, C.A., Lombardo, F., Dominy, B.W., and Feeney, P.J., Adv. Drug Del. Rev., 2001, vol. 46, p. 3. https://doi.org/10.1016/S0169-409X(00)00129-0

14. Ishikawa, N. and Kobayashi, Y., Fluorine Compounds, Chemistry and Application, Tokyo: Kodansha Scientific, 1979. 